\title{
Estratégia de crescimento clonal e fenologia de Syngonanthus chrysanthus Ruhland (Eriocaulaceae) nas baixadas entre dunas da Praia da Joaquina, Florianópolis, SC, Brasil ${ }^{1}$
}

\author{
Lúcia Helena Gütschow-Bento ${ }^{2,4}$, Tânia Tarabini Castellani², Benedito Cortês Lopes² e Péricles da Silva Godinhoº
}

Recebido em 15/12/2008. Aceito em 8/09/2009

\begin{abstract}
RESUMO - (Estratégia de crescimento clonal e fenologia de Syngonanthus chrysanthus Ruhland (Eriocaulaceae) nas baixadas entre dunas da Praia da Joaquina, Florianópolis, SC). Syngonanthus chrysanthus é uma planta que ocorre nos campos da restinga litorânea no sul e sudeste do Brasil. As baixadas entre dunas, heterogêneas no tempo e no espaço, variam quanto à disponibilidade hídrica, ora submetidas a alagamentos, ora a restrições hídricas, o que limita o recrutamento das espécies. Este trabalho avaliou aspectos fenológicos, ciclo de vida, potencial de reprodução sexuada e assexuada de $S$. chrysanthus como parte das estratégias de história de vida da espécie. Syngonanthus chrysanthus mostrou ser uma planta perene, potencialmente policárpica, com alta capacidade de reprodução vegetativa do tipo falangeal, o que permite seu estabelecimento em microambientes já colonizados. Syngonanthus chrysanthus também evidenciou recrutamento por plântulas e uma elevada produção de sementes que, dispersas ao longo de quase todo o ano, poderiam aumentar as chances de ocupar locais favoráveis ao seu estabelecimento, variáveis espacial e temporalmente nas baixadas entre dunas.

Palavras-chave: heterogeneidade ambiental, crescimento em falange, ciclo de vida, estruturas reprodutivas, restinga litorânea
\end{abstract}

\begin{abstract}
Clonal growth and phenology strategies of Syngonanthus chrysanthus Ruhland (Eriocaulaceae) in dune slacks at Joaquina Beach, Florianópolis, Santa Catarina state). Syngonanthus chrysanthus occurs on coastal plains in southern and southeastern Brazil. The dune slacks are heterogeneous in time and space and have variable water availability, sometimes flooded, sometimes dry, thus restricting species recruitment. This study examined phenological aspects, life cycle, sexual and asexual reproduction as part of the life history strategy of the species. Syngonanthus chrysanthus is a perennial plant, potentially polycarpic, with high capacity of vegetative reproduction of the phalanx type, which allows it to establish in micro-habitats already colonized. Syngonanthus chrysanthus also evidenced establishment by seedlings and high seed production. The seeds are dispersed over the entire year and may increase the opportunity of the species to grow in suitable habitats in the dune slacks.
\end{abstract}

Key words: habitat heterogeneity, phalanx type rhizomes, life cycle, reproductive structures, coastal plains

\section{Introdução}

As plantas podem apresentar reprodução sexuada e clonal mutuamente (Eckert 2002) e cada uma pode ser favorável sob diferentes condições ambientais (Prince \& Marshall 1999; Suzuki \& Stuefer 1999). Em ambientes adversos, usualmente pobres em nutrientes e submetidos à escassez hídrica, o estabelecimento e a sobrevivência de plântulas podem ser restritos, ficando limitados a curtos episódios de condições favoráveis no tempo e no espaço (Eriksson \& Fröborg 1996; Coelho et al. 2008a). Assim, nestes ambientes heterogêneos, a reprodução clonal representa uma alternativa para a persistência da população, bem como para a seleção, exploração e colonização de novos microambientes (Sampaio et al. 2004; 2005; Coelho et al. 2007; 2008a; b). A reprodução clonal prolonga a longevidade dos indivíduos da população quando os rametes permanecem unidos aos genetes e quando ocorre a formação de fragmentos independentes fisiologicamente, reduzindo os riscos de mortalidade dos genetes (Cook 1979; Callaghan 1987; Dong \& Alaten 1999). Além disso, os rizomas presentes em várias espécies clonais podem representar uma estrutura capaz de estocar recursos, como carboidratos, nutrientes minerais e água, podendo ser um meio de sobreviver à seca e ao fogo (Scatena et al. 1997; Cirne \& Scarano 2001; Coelho et al. 2007).
Diferentes formas clonais representam estratégias adaptativas em hábitats heterogêneos (Song \& Dong 2002). Quanto à disponibilidade e distribuição espacial de recursos, dois tipos de crescimento clonal são evidentes: falangeal, com conexões curtas entre os rametes, dispostos de forma compacta, expandindo-se lentamente e permanecendo na área por longos períodos, e guerrilha, com entrenós longos, de forma mais fragmentada, e com alta capacidade de penetração em novos hábitats, caracterizando um comportamento oportunista e agressivo (Lovett-Doust 1981). De uma forma geral, a estratégia compactada (falange) está associada a ambientes abertos, secos e pobres em nutrientes, enquanto que a fragmentada (guerrilha) a ambientes úmidos e ricos em nutrientes (van Groenendael et al. 1996). No entanto, ambas as estratégias evoluíram em ambientes com escassez hídrica e de nutrientes (Coelho et al. 2007; 2008a; b), sendo favorecidas de forma distinta em microambientes com diferentes graus de cobertura vegetal.

A reprodução clonal é muito comum em plantas (Olejniczak 2003) e se desenvolveu como uma alternativa à reprodução sexuada em muitas espécies de Eriocaulaceae (Coelho et al. 2006; 2007). Para essa família é observada a pseudoviviparidade, presente no gênero Leiothrix (Coelho et al. 2005; 2007; 2008a;b; Figueira \& Del Sarto 2007), e brotamento de rosetas a partir de rizomas, observado

\footnotetext{
1 Parte da Dissertação de Mestrado primeira Autora

2 Universidade Federal de Santa Catarina, Centro de Ciências Biológicas, Departamento de Ecologia e Zoologia, Laboratório de Ecologia Vegetal, Florianópolis, SC, Brasil

3 Universidade Federal de Santa Catarina, Centro de Ciências Biológicas, Departamento de Botânica, Florianópolis, SC, Brasil

4 Autor para correspondência: luciahelenagb@yahoo.com.br
} 
em espécies de Leiothrix (Coelho et al. 2007; 2008a;b) e Syngonanthus (Scatena et al. 1997; Schmidt et al. 2007). A pseudoviviparidade em Leiothrix tem sido caracterizada como uma estratégia do tipo guerrilha, enquanto que a rizomatosa, do tipo falangeal (Coelho et al. 2007; 2008a; b; Figueira \& Del Sarto 2007).

As plantas desta família, caracterizadas pelo hábito em roseta e inflorescência capituliforme (Moldenke \& Smith 1976), desenvolvem-se em diversos habitats, ocorrendo desde campos litorâneos da restinga até os campos do planalto (Reitz 1961), rupestres (Giulietti et al. 1996) e de altitude (Sano 2004). Nas restingas, onde o substrato arenoso possui baixos teores de nutrientes (Sampaio et al. 2005), as Eriocaulaceae ocorrem usualmente em baixadas entre dunas (Reitz 1961; Castellani et al. 1995). Esses ambientes são sujeitos a flutuações de umidade com períodos de restrição hídrica e de afloramento do lençol freático (Castellani et al. $1995 ; 2001)$, que em parte acarretam acentuadas variações na cobertura vegetal e composição florística ao longo do tempo (Castellani et al. 1995; Caetano 2003). Nas baixadas entre dunas, estudos de estratégia de vida de Eriocaulaceae foram realizados apenas para Actinocephalus polyanthus (Bong.) Sano, espécie não-clonal, cuja regeneração populacional depende de eventos de germinação maciça que ocorrem em anos com períodos de umidade favorável no outono (Castellani et al. 2001; Scherer \& Castellani 2004). Muitas plantas de duna florescem e frutificam em períodos de alta temperatura e pluviosidade, germinando no outono/inverno (Castellani et al. 1999). Estudos fenológicos com espécies de Syngonanthus foram realizados em campos rupestres, mostrando floração em períodos de baixa pluviosidade. A dispersão ocorre pelo vento (anemocórica) e pela queda dos escapos próximos ao genetes, coincidindo com a estação chuvosa, o que favorece o estabelecimento de plântulas através da germinação de sementes (Scatena et al. 1997; Schmidt et al. 2007; Cerqueira et al. 2008).

As Eriocaulaceae possuem grande interesse econômico referente à extração de capítulos (Giulietti et al. 1996) e escapos florais (Schmidt et al. 2007), sendo Syngonanthus o gênero mais comercializado (Miranda \& Giulietti 2001). Devido ao recurso econômico que as espécies desta família representam, vários estudos vêm sendo realizados a respeito de suas estratégias reprodutivas e fenologia (d'Eça-Neves \& Castellani 1994; Scatena et al. 1997; Coelho et al. 2005; 2006; 2007; 2008a; b; Ramos et al. 2005; Schmidt et al. 2007; Figueira \& Del Sarto 2007; Cerqueira et al. 2008). No litoral de Santa Catarina, plantas de Syngonanthus chrysanthus Ruhland deram origem a um cultivar ornamental comercializado na Europa (ANTHURA 2003), não havendo estudos sobre aspectos de sua fenologia e estratégias de reprodução.

Devido à heterogeneidade espacial e temporal dos ambientes de baixada entre dunas onde Syngonanthus chrysanthus ocorre e às variações de umidade que podem limitar o estabelecimento nestes ambientes, a avaliação de sua capacidade de reprodução clonal e se sua fenologia reprodutiva se ajusta às variações de fatores abióticos locais é importante para o entendimento das estratégias de vida de Eriocaulaceae em diferentes hábitats. Além disso, podem auxiliar no manejo desta espécie com exploração recente. O presente estudo abordou aspectos da fenologia reprodutiva, ciclo de vida e potencial de reprodução sexuada e assexuada em S. chrysanthus, de forma a responder: 1) Que fatores abióticos se relacionam ao desencadeamento das fenofases reprodutivas? 2) A espécie apresenta reprodução clonal como uma alternativa à sexuada? 3) Qual é o mecanismo de propagação vegetativa? 4) Como se caracterizam os indivíduos reprodutivos quanto ao tamanho, produção de capítulos e sementes?

\section{Materiais e métodos}

Espécie em estudo - Syngonanthus chrysanthus conhecida popularmente como "capipoatinga-dourada", é uma pequena erva rosetada de caule muito curto com folhas lineares com 20 a $55 \mathrm{~mm}$ de comprimento. Apresenta capítulos semi-globosos, podendo mostrar vários pedúnculos na mesma planta ou raramente um. Os pedúnculos podem ter de 12 a $25 \mathrm{~cm}$ de altura e os capítulos tem cerca de $5 \mathrm{~mm}$ de largura. As flores masculinas têm sépalas estreitas e espatuladas, pétalas alto-concrescidas e anteras exsertas. As flores femininas possuem sépalas semelhantes às masculinas, porém, com pétalas estreitas e glabras. A espécie é caracterizada como heliófita e seletiva higrófita, característica e exclusiva dos campos da restinga litorânea (Moldenke \& Smith 1976). Ocorre preferencialmente nos campos arenosos úmidos, em beira de lagoas e pequenas depressões e mais raramente nos banhados rasos, apresentando dispersão descontínua (Reitz 1961; Moldenke $\&$ Smith 1976). É uma espécie restrita à América do Sul (Giulietti \& Hensold 1990) e no Brasil, sua área de distribuição está compreendida entre Rio de Janeiro e Rio Grande do Sul (Moldenke \& Smith 1976).

Local de estudo - O presente estudo foi desenvolvido em áreas de baixada entre dunas, localizadas no Parque Municipal das Dunas da Lagoa da Conceição, litoral leste da Ilha de Santa Catarina, entre as latitudes $27^{\circ} 36^{\prime}$

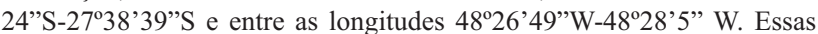
baixadas entre dunas se caracterizam por possuir cobertura vegetal predominantemente herbácea e pela ocorrência de alagamentos temporários (Castellani et al.1995).

Condições climáticas - A Ilha de Santa Catarina possui um clima mesotérmico úmido, tipo Cfa de Koeppen, com verões quentes e chuvas distribuídas ao longo de todo o ano. A temperatura média mensal é de $21^{\circ} \mathrm{C}$, variando de $16^{\circ} \mathrm{C}$ em julho a $25^{\circ} \mathrm{C}$ em fevereiro (CECCA 1997).

Um diagrama climático, adaptado de Walter (1986), foi elaborado com os valores médios de pluviosidade e temperatura média mensal de uma série temporal de 30 anos (1977-2006) obtidos da EPAGRI-SC (Fig.1a). Esse mostrou para a região de Florianópolis uma temperatura média anual de $20{ }^{\circ} \mathrm{C}$, oscilando entre $16^{\circ} \mathrm{C}$ e $18{ }^{\circ} \mathrm{C}$ no inverno e entre $24{ }^{\circ} \mathrm{C}$ e $26^{\circ} \mathrm{C}$ no verão. A média pluviométrica anual foi de $1738 \mathrm{~mm}$, com maiores índices em janeiro e fevereiro e menores índices de junho a agosto (Fig. 1a). O diagrama climático elaborado para o período de estudo (Fig. 1b) mostrou maior pluviosidade em 2005 (1697 mm) que em 2006 (1360 mm). Em 2005, o final do outono (maio - $222 \mathrm{~mm}$ ) e o período de agosto a setembro foram mais chuvosos, ocorrendo restrição hídrica em junho (32 mm). Em 2006, ocorreu um longo período com baixa pluviosidade do final de maio a setembro, com um mínimo de $31 \mathrm{~mm}$ em junho. Neste mesmo ano, os períodos úmidos e super-úmidos predominaram no verão (fevereiro - 261 $\mathrm{mm}$ ) e final da primavera (novembro $-368 \mathrm{~mm}$ ). As temperaturas médias anuais foram $21,1^{\circ} \mathrm{C}$ e $20,8^{\circ} \mathrm{C}$ para 2005 e 2006 , respectivamente, com médias mínimas entre $16,7^{\circ} \mathrm{C}$ e $14,9^{\circ} \mathrm{C}$ e médias máximas entre $24,6^{\circ} \mathrm{C}$ e $25,5^{\circ} \mathrm{C}$ (Fig. 1b). Quanto ao fotoperíodo (dados fornecidos pela EPAGRI$\mathrm{SC}$ ), as maiores médias mensais ocorreram na primavera e verão, sendo o maior registro em dezembro (14,73 h) (Fig. 2).

Fenologia reprodutiva - $\mathrm{O}$ registro foi realizado quinzenalmente entre novembro/2005 e dezembro/2006 (14 meses), numa área de baixada entre dunas 

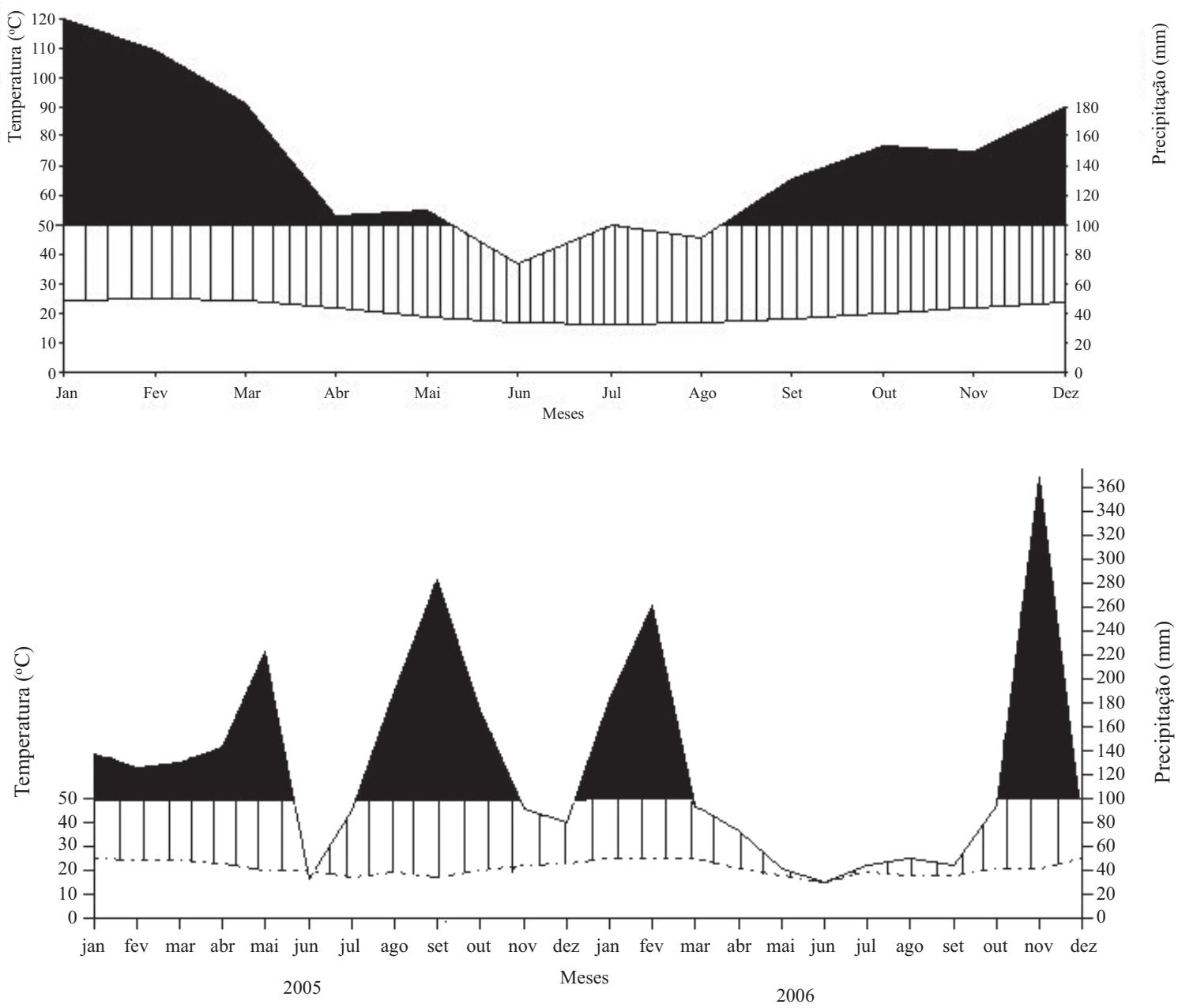

Figura 1. a. Dados climáticos para a Ilha de Santa Catarina, SC, Brasil. Temperaturas médias (linha tracejada) e pluviosidade (linha contínua) mensais para uma série temporal de 30 anos e b) diagrama climático com as médias mensais de temperatura (linha tracejada) e precipitação total (linha contínua) para os anos de 2005 e 2006, Florianópolis, SC. ( - período súper-úmido; \|- período úmido - déficit hídrico).

com expressiva ocorrência de $S$. chrysanthus. Oito quadrados permanentes de $30 \times 30 \mathrm{~cm}$, totalizando $0,72 \mathrm{~m}^{2}$, foram monitorados, estando estes distantes dois metros entre si e dispostos ao longo de duas transecções. Foi utilizado um quadrado de PVC ( $30 \times 30 \mathrm{~cm})$, subdividido em 36 quadrados por fios de nylon, para auxiliar na contagem das estruturas reprodutivas (inflorescência) em diferentes fenofases. Foi feita a contagem do número de capítulos por área ao invés do número de capítulos por roseta reprodutiva, devido à dificuldade de individualizar em campo as rosetas agrupadas em touceiras e identificar de quais rosetas provinham os escapos. O número de escapos na primeira observação (novembro de 2005) foi de 265, sendo as fenofases caracterizadas como: a) Emissão de escapo: a partir do momento em que os escapos florais começam a ser emitidos, com bainhas fechadas e capítulo incluso; b) Capítulo fechado: escapos completamente desenvolvidos, com bainhas abertas, capítulos expostos, porém envoltos completamente pelas brácteas; c) Capítulo aberto com flores em botão: escapos completamente desenvolvidos, com bainhas e capítulos abertos e flores em botão; d) Capítulo aberto em flor: escapos completamente desenvolvidos, com bainhas, capítulos e flores masculinas abertas, expondo suas anteras (as flores femininas são inconspícuas, sendo difícil sua visualização em campo); e) Frutos: escapos completamente desenvolvidos, com bainhas e capítulos abertos, com coloração escurecida e anteras não mais aparentes;. f) Dispersão: escapos completamente desenvolvidos, com bainhas e capítulos abertos, de coloração escurecida, com anteras não mais aparentes, começando a dispersar as sementes até a completa dispersão, restando apenas o escapo floral.

A floração da população foi caracterizada segundo os critérios propostos por Newstrom et al. (1994). Confeccionaram-se histogramas de freqüência para a avaliação da duração e intensidade de cada fenofase. Calculou-se a média de capítulos em cada fenofase para cada mês e estes dados foram correlacionados com a média mensal de temperatura, fotoperíodo e precipitação total dos meses de estudo através do índice de correlação de Spearman $\left(\mathrm{r}_{\mathrm{s}}\right)$ (Zar 1999), com uso do programa Bioestat 3.0 (Ayres et al. 2003). Correlações com temperatura e precipitação também foram feitas com os dados climáticos da série temporal de 30 anos.

Avaliação da mortalidade pós-reprodutiva - A verificação de mortalidade após os episódios reprodutivos foi realizada através do monitoramento mensal de indivíduos presentes em dois outros quadrados amostrais permanentes de $30 \times 30 \mathrm{~cm}$, localizados na área do acompanhamento fenológico. Em fevereiro de 2006, vinte indivíduos isolados ( $\mathrm{n}=20$ rosetas) e trinta indivíduos agrupados em cinco touceiras foram marcados com um palito etiquetado. No caso das cinco touceiras, todas as rosetas foram monitoradas. As avaliações foram realizadas até dezembro de 2006, após o final da dispersão.

Caracterização morfológica, capacidade de reprodução vegetativa e estimativa da produção de sementes - Em março, julho e novembro de 2006 foram extraídas, em três baixadas entre dunas, todas as rosetas $(n=467)$, tanto isoladas quanto agrupadas, presentes em quadrados amostrais de 10 $\mathrm{x} 10 \mathrm{~cm}$, sendo três quadrados por área, totalizando nove quadrados por data. O material foi examinado em laboratório, onde foi registrado: a) o número total de rosetas e touceiras por quadrado, b) o número de rosetas por touceiras (unidas através dos rizomas), c) o total de rosetas vegetativas e reprodutivas, d) o diâmetro basal de cada roseta, e) o número de folhas e o comprimento mínimo e máximo das folhas por roseta, f) o número 


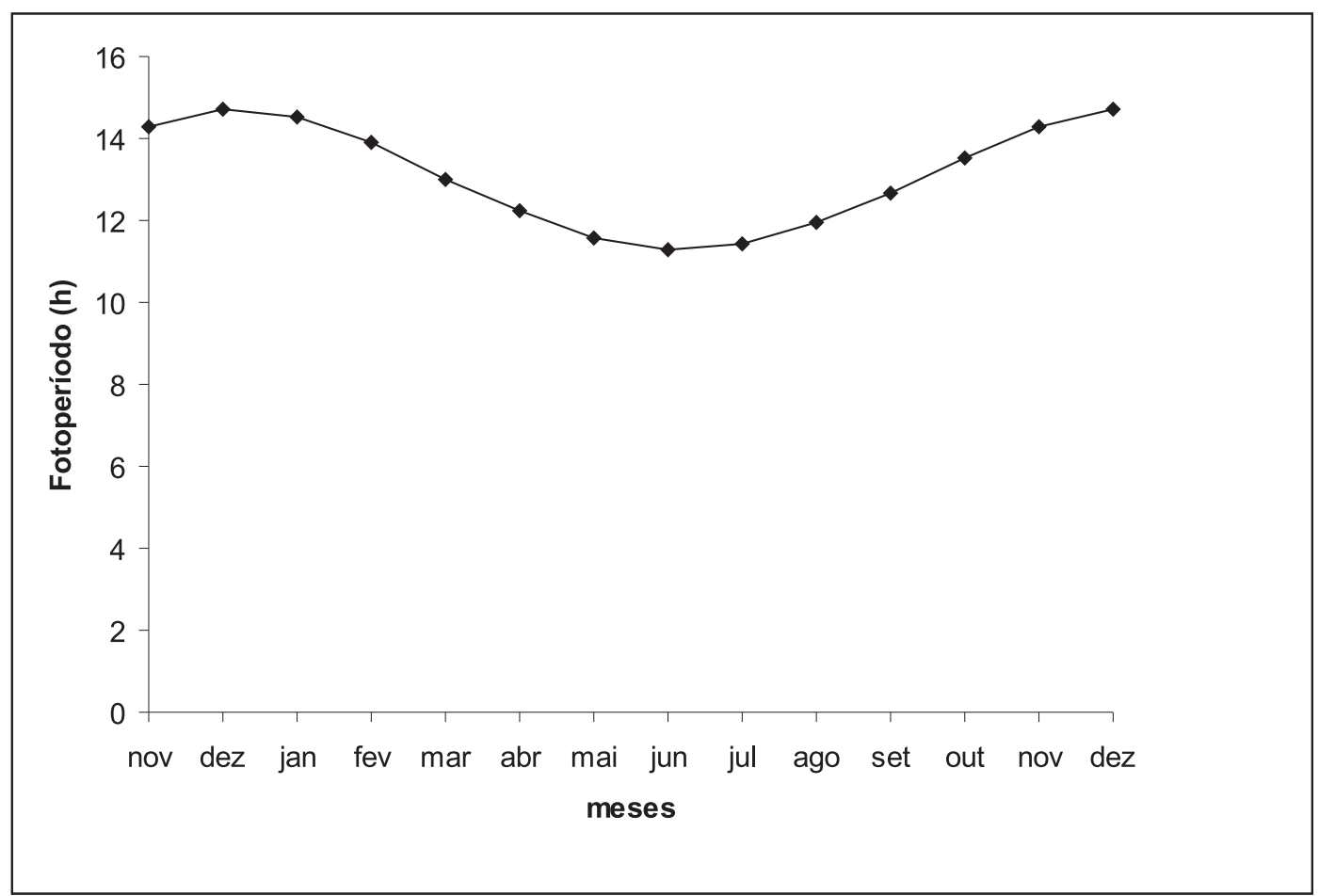

Figura 2. Fotoperíodo entre novembro de 2005 e dezembro de 2006 para Florianópolis, SC.

e comprimento dos escapos reprodutivos (considerando-se aqueles em capítulos fechados até fruto maduro) e g) o diâmetro dos capítulos. Para estimar a produção de sementes, 40 capítulos foram coletados em fase de fruto maduro e a contagem das sementes foi realizada em microscópio estereoscópico (Wild Leitz M3Z), com auxilio de um contador manual (Hope 8-004). Foi calculada a estimativa da produção média de sementes por roseta (número médio de capítulos/roseta x número médio de sementes/ capítulo) e por touceira (número estimado de sementes/roseta x número médio rosetas/touceira). Teste $\chi^{2}$ foi usado na avaliação da proporção de plantas reprodutivas nas diferentes datas amostrais e o teste de MannWhitney foi empregado na comparação do tamanho de rosetas de plantas reprodutivas e vegetativas (Zar 1999).

\section{Resultados}

Fenologia reprodutiva - O início do episódio reprodutivo de S. chrysanthus ocorreu no mês de agosto (Fig. 3a), com a emissão dos escapos florais. Este mês foi o mais expressivo quanto a esta fenofase $\left(\mathrm{n}=102 \mathrm{em} 0,72 \mathrm{~m}^{2}\right)$, que se estendeu até novembro. Também em agosto, as bainhas dos escapos de algumas inflorescências começaram a abrir, expondo os capítulos fechados. Setembro $(n=323)$ e outubro $(n=341)$ foram os meses de maior intensidade desta fenofase, que se prolongou até abril (Fig. 3b). Entre fevereiro e abril, foi registrado apenas um pequeno número de capítulos fechados, que não se desenvolveram. Em setembro, iniciou-se a abertura dos capítulos, com flores em fase de botão. Esta fase ocorreu até fevereiro com picos de maior intensidade em outubro e novembro (Fig. 3c). Em outubro, as flores masculinas começaram a abrir (Fig. 3d). O final da antese floral ocorreu em fevereiro. Em 2005, o pico de intensidade foi mais tardio, ocorrendo em dezembro $(n=225)$, enquan- to que em 2006 foi em novembro $(n=295)$. No mês de dezembro, muitos capítulos frutificaram. A época de maior intensidade de frutificação foi mais tardia no primeiro ciclo reprodutivo, ocorrendo em janeiro $(\mathrm{n}=200)$. No segundo ciclo, esta ocorreu em dezembro, último mês de observação, com 310 capítulos (Fig. 3e). A fase de frutos em dispersão foi a mais longa, compreendida entre janeiro e outubro. Os picos de dispersão foram em fevereiro $(n=170)$ e março $(\mathrm{n}=182)$ (Fig. 3f).

A fenofase capítulos abertos com flores em botão apresentou correlação positiva significativa com o fotoperíodo $\left(\mathrm{r}_{\mathrm{s}}=0,79 ; \mathrm{p}<0,05\right)$. Tanto o início (setembro) quanto os picos (outubro e novembro) desta fenofase ocorreram em meses com maior comprimento do dia. Os capítulos abertos em flor também mostraram correlação positiva significativa com o fotoperíodo $\left(\mathrm{r}_{\mathrm{s}}=0,84 ; \mathrm{p}<0,05\right)$, assim como com a precipitação $\left(\mathrm{r}_{\mathrm{s}}=0,74 ; \mathrm{p}<0,05\right)$, com o início e os picos de floração ocorrendo em meses de alta pluviosidade. Os frutos apresentaram correlação positiva significativa com a temperatura $\left(r_{s}=0,73 ; p<0,05\right)$. Tanto o início (dezembro) quanto os picos (dezembro e janeiro) ocorreram em meses de maior temperatura $\left(25^{\circ} \mathrm{C}\right)$ e fotoperíodo $\left(\mathrm{r}_{\mathrm{s}}=0,84 ; \mathrm{p}<0,05\right)$.

As análises de correlação feitas com a série temporal de 30 anos mostraram que os capítulos abertos em botão apresentaram correlação significativa e positiva com a precipitação $\left(r_{s}=0,62 ; p<0,05\right)$. As fenofases de floração e frutificação mostraram correlação significativa e positiva com a precipitação $\left(r_{s}=0,71 ; p<0,05\right.$ e $r_{s}=0,61 ; p<0,05$, respectivamente) e com a temperatura $\left(r_{s}=0,62 ; p<0,05\right.$ e 
Acta bot. bras. 24(1): 205-213. 2010 .

209
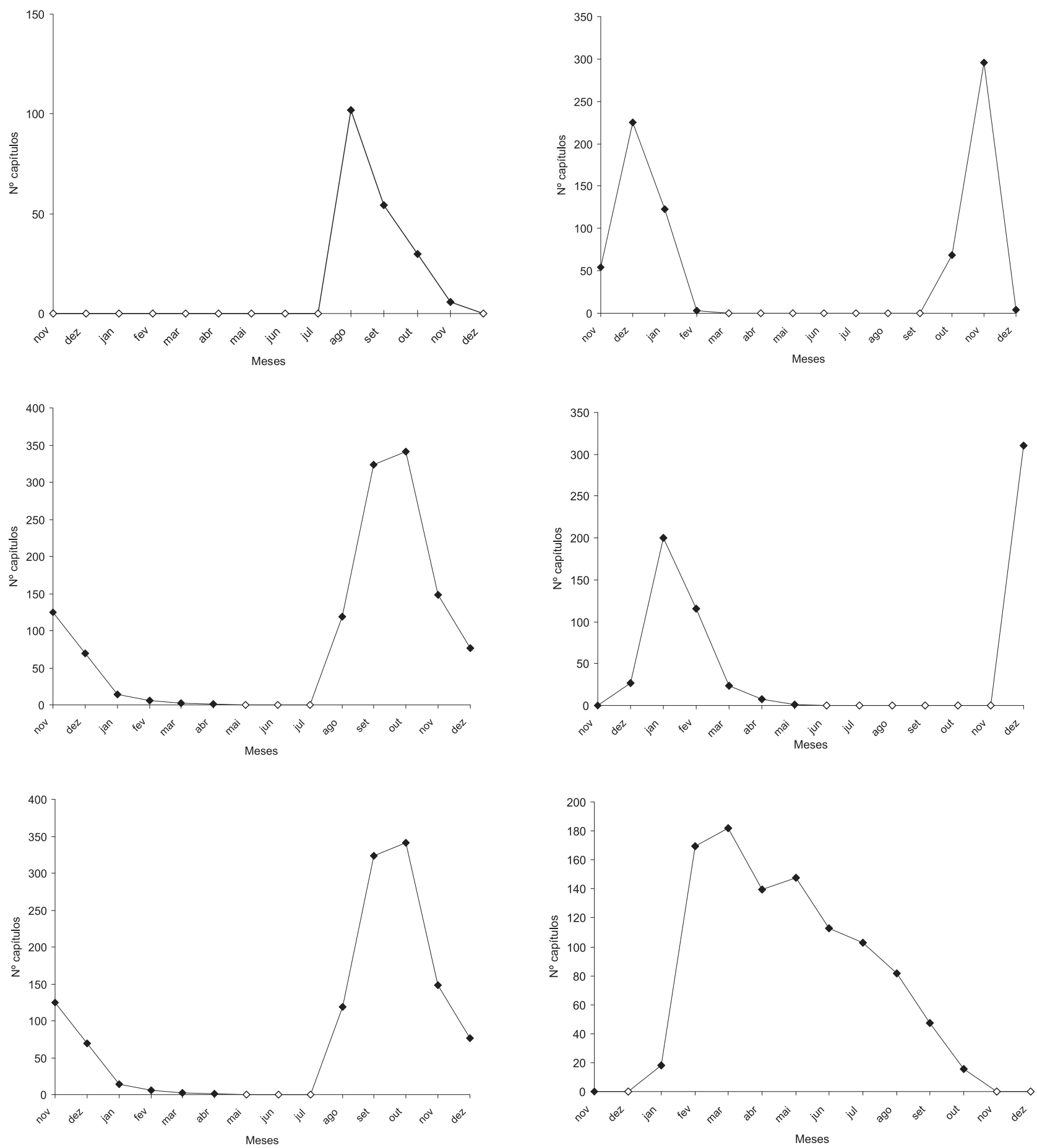

Figura 3. Número total de capítulos de Syngonanthus chrysanthus em diferentes fenofases a) emissão de escapos; b) capítulos fechados; c) capítulos abertos com flores em botão; d) capítulos abertos em flor masculina; e) capítulos em fruto e f) capítulos em dispersão de sementes, em 0,72 m², de novembro de 2005 e dezembro de 2006, em baixada úmida das dunas da Praia da Joaquina, Florianópolis, SC ( $\square$ - ausência de fenofase).

$\left.r_{s}=0,68 ; p<0,05\right)$. Não foram feitas correlações com séries temporais de fotoperíodo.

Avaliação da mortalidade pós-reprodutiva - Não foram visualizados sinais de senescência após a reprodução nas plantas avaliadas em dezembro de 2006. Dentre as 20 rosetas isoladas marcadas em fevereiro de 2006, 11 floresceram e nenhuma morreu até dezembro de 2006. As nove rosetas restantes também sobreviveram e permaneceram vegetativas. Dentre as 30 rosetas agrupadas em touceiras, 28 se reproduziram, não havendo sinais de senescência após este período de 11 meses. Nessas touceiras monitoradas, houve brotamento de 40 novas rosetas no período.

Caracterização morfológica, capacidade de reprodução vegetativa e estimativa da produção de sementes - O número 
de rosetas coletadas e analisadas em laboratório para a caracterização da capacidade reprodutiva foi de 222, 138 e 107 rosetas por período, nas três áreas. Foi registrado um total de $368(79,3 \%)$ rosetas agrupadas em 53 touceiras, contendo cerca de 6,9 rosetas cada (Tab. 1). Em cada agrupamento, todas as rosetas estavam ligadas através de um curto rizoma. Noventa e nove $(20,7 \%)$ rosetas eram isoladas e sem vestígios de ligações por rizomas, indicando estabelecimento via reprodução sexuada (Tab. 1).

Em março de 2006, as porcentagens médias de indivíduos vegetativos foram maiores $(83,3 \%)$ que de plantas reprodutivas $(16,6 \%)$ (Tab. 1, $\left.\chi^{2}=98,7 ; p<0,05\right)$. Em julho de 2006 não houve diferença, com $55,1 \%$ de indivíduos vegetativos e $44,9 \%$ reprodutivos $\left(\chi^{2}=0,23 ; p>0,05\right)$. Em novembro de 2006 , as plantas reprodutivas foram mais freqüentes $(91,6 \%)$ que vegetativas $(8,4 \%)\left(\chi^{2}=74,0 ; p<0,05\right)$.

Houve diferença significativa $(\mathrm{U}=18948,50 ; \mathrm{p}<$ $0,0001)$ entre o diâmetro médio das rosetas vegetativas e reprodutivas, sendo essas menores (Tab. 2). As rosetas reprodutivas apresentaram cerca de dois escapos florais (Tab. 2), estimando-se 13,8 escapos florais por touceira. O número de sementes por capítulo $(\mathrm{n}=40)$ variou de 61 a 825 , sendo em média 495,8 (d.p. $=175$ ). A produção de sementes por roseta foi estimada em 991,6 sementes, e por touceira, em 3421,02 sementes.

\section{Discussão}

Apesar de o presente estudo acompanhar a fenologia de S. chrysanthus por apenas 14 meses, o comportamento ora encontrado sugere um início de período reprodutivo, emissão de escapos e capítulos fechados, em época de menor pluviosidade para a região de Florianópolis. A antese floral e a maturação de frutos, porém, estiveram relacionadas a períodos com elevação de pluviosidade, temperatura e fotoperíodo. O maior número de capítulos em dispersão ocorreu no final de verão e início de outono, sendo essas sementes lançadas ao solo sob condições favoráveis de umidade. Em ambientes de dunas, com baixa capacidade de retenção hídrica no solo, tem sido sugerido que o outono é uma época mais propícia para a germinação, com temperaturas mais amenas e menor evapotranspiração (Ernst 1985; Roozijn \& Andel 1985; Castellani et al. 1999). No entanto, a duração da fase de dispersão de $S$. chrysanthus é longa e não se restringe aos períodos de maior pluviosidade. Essa espécie coloniza um hábitat de duna muito variável quanto à disponibilidade hídrica, temporal e espacialmente, ora sujeita à restrição de umidade ora sujeita a alagamento. Neste contexto, sugerimos que a longa fenofase de dispersão, aliada ao elevado número de sementes produzidas, podem ser vantajosos em termos ecológicos, já que a liberação contínua de sementes aumentaria a chance de encontro de condições favoráveis de germinação. Outros estudos sugerem que, para espécies ocorrentes nestes hábitats, seja favorável um eficiente mecanismo de dispersão, assim como um banco de sementes permanente no solo de grande longevidade (Schat \& Scholten 1985; Castellani et al. 1995; Looney \& Gibson 1995).

O comportamento fenológico de S. chrysanthus difere de outras espécies do gênero, que ocorrem em formações vegetais sujeitas a uma estação seca mais definida. Syngonanthus elegans Ruhland na Serra do Cipó, MG, (Scatena et al. 1997) e $S$. nitens (Bong.) Ruhland no Jalapão, TO, (Schmidt et al. 2007) apresentam predominância de capítulos

Tabela 1. Número de rosetas de Syngonanthus chrysanthus coletadas em quadrados de $10 \times 10 \mathrm{~cm}$ em março, julho e novembro de 2006 (n $=9$ por período) em baixadas úmidas entre dunas na Praia da Joaquina, Florianópolis, SC. A proporção de rosetas isoladas e agrupadas e de rosetas reprodutivas e vegetativas é mostrada, assim como os números médios ( \pm desvio padrão), mínimos e máximos de rosetas por touceira.

\begin{tabular}{|c|c|c|c|c|c|c|c|}
\hline & Total & Isoladas & Agrupadas & Touceiras & Rosetas/touceira & Veg. & Rep. \\
\hline Mar. & 222 & $49(22 \%)$ & $\begin{array}{c}173 \\
(78 \%)\end{array}$ & 12 & $\begin{array}{c}14,4( \pm 10,9) \\
(2-32)\end{array}$ & $\begin{array}{c}185 \\
(83 \%)\end{array}$ & $\begin{array}{c}37 \\
(17 \%)\end{array}$ \\
\hline Jul. & 138 & $28(20 \%)$ & $\begin{array}{c}110 \\
(80 \%)\end{array}$ & 23 & $\begin{array}{c}4,8( \pm 3,4) \\
(2-17)\end{array}$ & $\begin{array}{c}76 \\
(55 \%)\end{array}$ & $\begin{array}{c}62 \\
(45 \%)\end{array}$ \\
\hline Nov. & 107 & $22(21 \%)$ & $\begin{array}{c}85 \\
(79 \%)\end{array}$ & 18 & $\begin{array}{c}4,7( \pm 3,0) \\
\quad(2-11)\end{array}$ & $\begin{array}{c}9 \\
(8 \%)\end{array}$ & $\begin{array}{c}98 \\
(92 \%)\end{array}$ \\
\hline Total & 467 & $99(21 \%)$ & $\begin{array}{c}368 \\
(79 \%)\end{array}$ & 53 & $\begin{array}{c}6,9( \pm 7,0) \\
(2-32)\end{array}$ & $\begin{array}{c}270 \\
(58 \%)\end{array}$ & $\begin{array}{c}197 \\
(42 \%)\end{array}$ \\
\hline
\end{tabular}

Tabela 2. Média, desvio padrão, mínimo e máximo do diâmetro de rosetas vegetativas e reprodutivas, número e comprimento da folha (menor e maior), número de escapos por roseta, altura dos escapos e diâmetro de capítulos abertos de Syngonanthus chrysanthus em baixada entre dunas na Praia da Joaquina, Florianópolis, SC.

\begin{tabular}{|c|c|c|c|c|c|c|c|c|}
\hline & \multicolumn{2}{|c|}{ Diâmetro de roseta $(\mathrm{cm})$} & \multirow{2}{*}{$\begin{array}{c}\text { Folhas/ } \\
\text { roseta }\end{array}$} & \multicolumn{2}{|c|}{ Comprimento da folha $(\mathrm{cm})$} & \multirow{2}{*}{$\begin{array}{l}\text { Escapos/ } \\
\text { roseta }\end{array}$} & \multirow{2}{*}{$\begin{array}{l}\text { Altura dos escapos } \\
(\mathrm{cm})\end{array}$} & \multirow{2}{*}{$\begin{array}{l}\text { Diâmetro dos } \\
\text { capítulos }(\mathrm{cm})\end{array}$} \\
\hline & Veg. & Rep. & & menor & maior & & & \\
\hline Média & $4,6(n=281)$ & $\begin{array}{c}3,9 \\
(n=186)\end{array}$ & $\begin{array}{c}43 \\
(n=467)\end{array}$ & $\begin{array}{c}0,37 \\
(n=467)\end{array}$ & $\begin{array}{c}2,5 \\
(n=467)\end{array}$ & $\begin{array}{c}2,0 \\
(\mathrm{n}=186)\end{array}$ & $\begin{array}{c}9,5 \\
(n=177)\end{array}$ & $\begin{array}{c}0,3 \\
(n=177)\end{array}$ \\
\hline d.p. & 1,6 & 1,3 & 24,2 & 0,2 & 1,0 & 1,2 & 2,2 & 0,1 \\
\hline Mín. & 1 & 0,5 & 3 & 0,1 & 0,4 & 1,0 & 2,5 & 0,3 \\
\hline Máx. & 9 & 8 & 179 & 1,8 & 5,6 & 6,0 & 14,7 & 1,0 \\
\hline
\end{tabular}


em flor no período de escassez hídrica. Syngonanthus тисиgensis Giul. subsp. mucugensis e $S$. curralensis Moldenke mostram separação temporal de crescimento vegetativo no período chuvoso e presença de estruturas reprodutivas no período seco, em campos rupestres na Chapada Diamantina, BA, (Cerqueira et al. 2008). Nas espécies acima citadas, a dispersão de sementes tende a ocorrer no início do período úmido, o que auxiliaria na germinação de sementes que caem com os escapos próximos à planta-mãe ou são dispersas pelo vento. Diversos comportamentos fenológicos foram encontrados para as espécies de Eriocaulaceae nos campos rupestres onde essas foram mais estudadas. Actinocephalus polyanthus tem o início reprodutivo coincidente com o início da estação chuvosa (Sano 1996) e Leiothrix curvifolia var. lanuginosa (Bong.) Ruhland e L. crassifolia (Bong.) Ruhland apresentam florescimento correlacionado à disponibilidade de água no solo (Coelho et al. 2008a). Foi sugerido que a seca sazonal pode ter favorecido a sincronia da floração, de modo que uma intensa produção de capítulos florais durante a estação chuvosa permitiria a dispersão de sementes em um solo ainda úmido, favorecendo o estabelecimento de plântulas (Coelho et al. 2008a).

A floração de $S$. chrysanthus parece ser anual, regular, primaveril e com duração intermediária (senso Newstron et al. 1994). Uma duração intermediária também foi relatada para S. mucugensis e S. curralensis (Cerqueira et al. 2008). Essas, no entanto, floresceram por no máximo três meses e $S$. chrysanthus por cinco meses. Syngonanthus chrysanthus diferiu do observado para $S$. elegans e $S$. nitens, que mostraram duração longa (seis meses) e curta (dois meses), respectivamente (Scatena et al. 1997; Schmidt et al. 2007). Metodologias distintas para a avaliação da floração dificultam, no entanto, comparações entre pesquisas com espécies do mesmo gênero. No presente estudo avaliou-se a antese das flores dentro da inflorescência, e não a abertura do capítulo floral para caracterizar a floração (Scatena et al. 1997; Schmidt et al. 2007). Tal diferença metodológica indica que a floração de $S$. chrysanthus é mais longa que o aqui reportado, o que permite sugerir que há maiores restrições ambientais para a floração de espécies do gênero que ocorrem em ambientes mais sazonais. Em relação à frutificação e dispersão, a duração em $S$. chrysanthus foi superior ao registrado para outras espécies do gênero (Scatena et al. 1997; Schmidt et al. 2007; Cerqueira et al. 2008), o que pode representar uma estratégia para aumentar as chances de germinação das sementes em um ambiente com muita variabilidade de umidade.

Sugere-se que $S$. chrysanthus possui ciclo de vida perene, com sobrevivência pós-reprodutiva, o que possibilitaria policarpia. Esta observação é reforçada através do mapeamento e análise de rosetas feito para um estudo demográfico, que mostrou capacidade de rosetas se reproduzirem em anos subseqüentes (Gütschow-Bento, dados não publicados). Espécies policárpicas tendem a alocar menos recursos para a reprodução do que as monocárpicas e, além disso, podem apresentar várias gerações sobrepostas (Begon et al. 1988). Em $S$. chrysanthus foram observadas rosetas reprodutivas em várias classes de diâmetro, diferente do descrito para a monocárpica $A$. polyanthus (Castellani et al. 2001), onde a chance de reprodução aumentava com o tamanho da planta. Um tamanho mínimo para se reproduzir também foi registrado para outras Eriocaulaceae, como L. flagellaris Ruhland (Figueira \& Del Sarto 2007), L. curvifolia var. lanuginosa e L. crassifolia (Coelho et al. 2007). Talvez essa relação não tenha sido encontrada para $S$. chrysanthus, devido ao crescimento clonal em touceiras. Uma touceira pode ser composta somente por rosetas vegetativas, reprodutivas ou ambas. Desta forma, rosetas reprodutivas pertencentes a um mesmo genete podem ter seu diâmetro influenciado pela interferência das demais, devendo ser considerada a integridade fisiológica do genete (Cook 1979; Watkinson $\&$ White 1985). No entanto, o monitoramento de rosetas isoladas oriundas de germinação de Leiothrix curvifolia e L. crassifolia mostrou que estas também formam clones compactos e apresentam um tamanho mínimo de roseta para ocorrência de reprodução (Coelho et al. 2007).

Syngonanthus chrysanthus apresentou menor proporção de plantas isoladas quando comparadas àquelas conectadas por rizomas, sugerindo um favorecimento do crescimento clonal. Essa estratégia é referida como vantajosa para a ocupação de ambientes com recursos limitados e heterogêneos, como dunas e restingas (Dong \& Alaten 1999; Sampaio et al. 2004; 2005; Castellani \& Santos 2006) e campos rupestres (Coelho et al. 2008a). Em dunas, a disponibilidade de água e nutrientes é limitada e variável e o crescimento clonal será proporcional a estas limitações (Dong \& Alaten 1999), sendo favorável para espécies com crescimento falangeal (van Groenendael et al. 1996), como descrito para S. chrysanthus. Em ambientes heterogêneos de restinga, a produção de rametes pode também diferir em função da cobertura vegetal associada e um crescimento clonal direcional pode selecionar hábitats favoráveis ao desenvolvimento dos rametes, escapando da interferência inter-específica (Sampaio et al. 2004; 2005).

Provavelmente, os microambientes de baixadas, onde ocorre S. chrysanthus (Gütschow-Bento et al. 2006), acumulam mais nutrientes em comparação com áreas mais elevadas de dunas. É comprovado que uma maior concentração de nutrientes induz a uma diminuição no comprimento dos entre-nós e aumento na produção de rametes, tendendo a uma arquitetura falangeal (Slade \& Hutchings 1987), característica de espécies clonais estoloníferas e/ou rizomatozas (Callaghan 1987), como parece ser S. chrysanthus. Este comportamento representa uma estratégia de consolidação e ocupação de hábitats, promovendo a permanência dos clones em ambientes favoráveis ao seu estabelecimento (Kroon \& Hutchings 1995).

Scatena et al. (1997) observaram a presença de amido armazenado nos rizomas de S. elegans, sendo citada como uma adaptação das Eriocaulaceae em ambientes que apresentam 
escassez hídrica temporária. Sugere-se que seja estudada a presença de amido em S. chrysanthus, já que essa seria uma característica favorável para a manutenção das populações no ambiente de dunas.

Com base nos resultados obtidos, podemos sugerir que $S$. chrysanthus possui ampla capacidade de crescimento clonal do tipo falangeal através de rizomas curtos, caracterizando um comportamento "forrageador", permitindo seu estabelecimento e permanência em microambientes já colonizados. Como demais espécies clonais (Eriksson 1989; Castellani \& Santos 2006; Coelho et al. 2008a; b), S. chrysanthus também evidencia estabelecimento de plântulas por sementes em áreas já colonizadas. Uma elevada produção de sementes, dispersas ao longo de quase todo o ano, talvez represente uma estratégia que amplie as chances da espécie encontrar condições favoráveis para germinação e desenvolvimento em um ambiente tão variável como nas baixadas entre dunas.

\section{Agradecimentos}

A Nivaldo Peroni e Adelar Mantovani pelas sugestões dadas na primeira versão deste trabalho e a três revisores anônimos que contribuíram para a versão final do manuscrito. Aos estagiários do Laboratório de Ecologia Vegetal ECZ/CCB/UFSC pela ajuda em campo, à CAPES pela concessão de bolsa a L.H. Gütschow-Bento e à SESU/MEC pela bolsa do programa PET à T.T. Castellani. À EPAGRI-SC por disponibilizar os dados climatológicos e ao Departamento de Ecologia e Zoologia ECZ/CCB/UFSC pelo apoio logístico.

\section{Referências bibliográficas}

Ayres, M.; Ayres, M., Jr.; Ayres, D.L. \& Santos, A.A.S. 2003. BioEstat 3.0: aplicações estatísticas nas áreas das ciências bio-médicas. Belém, Sociedade Civil Mamirauá/MCT-CNPq/Conservation International.

ANTHURA 2003. Cultivation Guidelines of Syngonanthus chrysanthus "Mikado". http://www.ecaa.ntu.edu.tw/weifang/lab551/flower/phal/ anthura_pot/manmikuk.pdf.

Begon, M.; Harper, J.L. \& Townsend, C.R. 1988. Ecologia: Indivíduos, poblaciones y comunidades. Barcelona, Omega.

Caetano, V.L. 2003. Dinâmica sazonal e fitossociologia da vegetação herbácea de uma baixada úmida entre dunas, Palmares do Sul, Rio Grande do Sul, Brasil. Iheringia, Série Botânica 58: 81-102.

Callaghan, T.V. 1987. Physiological and demographic implications of modular construction in cold environments. Pp. 111-135. In: A.J. Davy; M.J. Hutchings \& A.R. Watkinson (eds.). Plant Population Ecology. Oxford, Blackwell Scientific Publications.

Castellani, T.T.; Caus, C.A. \& Vieira, S. 1999. Fenologia de uma comunidade de duna frontal no sul do Brasil. Acta Botanica Brasilica 13: 99-113.

Castellani, T.T., Folchini, R. \& Scherer, K.Z. 1995. Variação temporal da vegetação em um trecho de baixada úmida entre dunas, Praia da Joaquina, Florianópolis, SC. Insula 24: 37-70.

Castellani, T.T. \& Santos, F.A.M. 2006. Abundância, sobrevivência e crescimento de plântulas de Ipomoea pes-caprae (L.) R. Br. na Ilha de Santa Catarina, SC, Brasil. Acta Botanica Brasilica 20: 875-885.

Castellani, T.T., Scherer, K.Z. \& Paula, G.S. 2001. Population ecology of Paepalanthus polyanthus (Bong.) Kunth: demography and life history of a sand dune monocarpic plant. Revista Brasileira de Botânica 24: $123-134$.

CECCA. 1997. Unidades de conservação e áreas protegidas da Ilha de Santa Catarina. Florianópolis, Insular.

Cerqueira, C.O., Funch, L.S. \& Borba, E.L. 2008. Fenologia de Syngonanthus mucugensis Giul. subsp. mucugensis e $S$. curralensis Moldenke (Eriocaulaceae), nos municípios de Mucugê e Morro do Chapéu, Chapada Diamantina, BA, Brasil. Acta Botanica Brasilica 22: $962-969$.
Cirne, P. \& Scarano, F.R. 2001. Resprouting and growth dynamics after fire of the clonal shrub Andira legalis (Leguminosae) in a sandy coastal plain in south-eastern Brazil. Journal of Ecology 89: 351-357.

Coelho, F.F.; Capelo, C.; Neves, A.C.O.; Capelo, C. \& Figueira, J.E.C. 2005. Pseudovivipary in two rupestrian endemic species (Leiothrix spiralis and Leiothrix vivipara). Current Science 88: 1225-1226.

Coelho, F.F; Capelo, C.; Neves, A.C.O.; Capelo, C.; Martins, R. \& Figueira, J.E.C. 2006. Seasonal timing of pseudoviviparous reproduction of Leiothrix (Eriocaulaceae) rupestrian species in south-eastern Brazil. Annals of Botany 98: 1189-1195.

Coelho, F.F; Capelo, C.D.L.; Neves, A.C.O. \& Figueira, J.E.C. 2007. Vegetative propagation strategies of four rupestrian species of Leiothrix (Eriocaulaceae). Revista Brasileira de Botânica 30: 687-694.

Coelho, F.F.; Capelo, C. \& Figueira, J.E.C. 2008a. Seedlings and ramets recruitment in two rhizomatous species of Rupestrian grasslands: Leiothrix curvifolia var. lanuginosa and Leiothrix crassifolia (Eriocaulaceae). Flora 203: 152-161.

Coelho, F.F.; Capelo, C.; Ribeiro, L.C. \& Figueira, J.E.C. 2008b Reproductive modes in Leiothrix (Eriocaulaceae) in south-eastern Brazil: the role of microenvironmental heterogeneity. Annals of Botany 101: 353-360.

Cook, R.E. 1979. Asexual reproduction: a further consideration. American Naturalist 113: 769-772.

d'Eça-Neves, F.F. \& Castellani, TT. 1994. Fenologia e aspectos reprodutivos de Paepalanthus polyanthus (Bong.) Kunth (Eriocaulaceae) em baixada úmida entre dunas na Praia da Joaquina, Ilha de Santa Catarina, SC. Insula 23: 121-149.

Dong, M. \& Alaten, B. 1999. Clonal plasticity in response to rhizome severing and heterogeneous resource supply in the rhizomatous grass Psammochloa villosa in an Inner Mongolian dune, China. Plant Ecology 141: 53-58.

Eckert, C.G. 2002. The loss of sex in clonal plants. Evolutionary Ecology 15: $501-520$

Eriksson, O. 1989. Seedling dynamics and life histories in clonal plants. Oikos 55: 231-238.

Eriksson, O. \& Fröborg, H. 1996. "Windows of opportunity" for recruitment in long-lived clonal plants: experimental studies of seedling establishment in Vaccinium shrubs. Canadian Journal of Botany 74: 1369-1374.

Ernst, W.H.O. 1985. Some considerations of and perspectives in coastal ecology. Vegetatio 62: 533-545.

Figueira, J.E. \& Del Sarto, M.C.L. 2007. Clonal growth and dispersal potential of Leiothrix flagellaris Ruhland (Eriocaulaceae) in the rocky grasslands of Southeastern Brazil. Revista Brasileira de Botânica 30: 679-686.

Giulietti, A.M \& Hensold, N. 1990. Padrões de distribuição geográfica dos gêneros de Eriocaulaceae. Acta Botanica Brasilica 4: 133-159.

Giulietti, A.M.; Wanderley, M.G.L.; Longhi-Wagner, H.M.L.; Pirani, J.R. \& Parra, L.R. 1996. Estudos em "sempre-vivas": Taxonomia com ênfase nas espécies de Minas Gerais, Brasil. Acta Botanica Brasilica 10: $329-377$

Gütschow-Bento, L.H.; Castellani, T.T.; Godinho, P.S.; Cruz, L.; Reis, M. \& Guimarães, T.B. 2006. Caracterização populacional de Syngonanthus chrysanthus Ruhland (Eriocaulaceae), no Parque Municipal das dunas da Lagoa da Conceição, Florianópolis, SC. Pp. 1-4. In: Encontro nacional de gerenciamento costeiro (ENCOGERCO). Florianópolis, Agência Brasileira de Gerenciamento Costeiro.

Kroon, H. \& Hutchings, M.J. 1995. Morphological plasticity in clonal plants: the foraging concept reconsidered. Journal of Ecology 83: 143-152.

Looney, P.B. \& Gibson, D.J. 1995. The relationship between the soil seed bank and above-ground vegetation of a coastal barrier island. Journal of Vegetation Science 6: 825-836.

Lovett-Doust, L. 1981. Population dynamics and local specialization in a clonal perennial (Ranunculus repens). I. The dynamics of ramets in contrasting habitats. Journal of Ecology 69: 743-755.

Miranda, E.B \& Giulietti, A.M. 2001. Eriocaulaceae no Morro Pai Inácio (Palmeiras) e Serra da Chapada Diamantina (Lençóis), Chapada Diamantina, Bahia, Brasil. Sitientibus Série Ciências Biológicas 1: $15-32$. 
Moldenke, H.N. \& Smith, L.B. 1976. Eriocauláceas. Pp. 2-103. In: R. Reitz (ed). Flora Ilustrada Catarinense. Itajaí, Herbário Barbosa Rodrigues.

Newstron, L.E., Frankie, G.W. \& Baker, H.G. 1994. A new classification for plant phenology based on flowering patterns in lowland tropical rain forest trees at La Selva, Costa Rica. Biotropica 26: 141-159.

Olejniczak, P. 2003. Optimal allocation to vegetative and sexual reproduction in plants: the effect of ramet density. Evolutionary Ecology 17: 265-275.

Prince, E.A.C. \& Marshall, C. 1999. Clonal plants and environment heterogeneity. Plant Ecology 141: 3-7.

Ramos, C.O.C.; Borba, E.L. \& Funch, L.S. 2005. Pollination in Brazilian Syngonanthus (Eriocaulaceae) species: evidence for entomophily instead of anemophily. Annals of Botany 96: 387-397.

Reitz, R. 1961. Vegetação da zona marítima de Santa Catarina. Sellowia 13: $17-115$.

Sampaio, M.C.; Araújo, T.F.; Scarano, F.R. \& Stuefer, J.F. 2004. Directional growth of a clonal bromeliad species in response to spatial habitat heterogeneity. Evolutionary Ecology 18: 429-442.

Sampaio, M.C.; Picó, F.X. \& Scarano, F.R. 2005. Ramet demography of a nurse bromeliad in Brazilian restingas. American Journal of Botany 92: 674-681.

Sano, P.T. 1996. Fenologia de Paepalanthus hilairei Koern., P. polyanthus (Bong.) Kunth e P. robustus Silveira: Paepalanthus sect. Actinocephalus Koern. (Eriocaulaceae). Acta Botanica Brasilica 10: $317-328$.

Sano, P.T. 2004. Actinocephalus (Körn.) Sano (Paepalanthus sect. Actinocephalus), a new genus of Eriocaulaceae, and other taxonomic and nomenclatural changes involving Paepalanthus Mart. Taxon 53: 99-107.

Scatena, V.L.; Lima, A.M.A. \& Filho, J.P.L. 1997. Aspectos fenológicos de Syngonanthus elegans (Bong.) Ruhl. (Eriocaulaceae) da serra do Cipó, MG, Brasil. Arquivos de Biologia \& Tecnologia 40: 153-167.

Schat, H. \& Scholten, M. 1985. Comparative population ecology of dune slack species: the relation between population stability and germination behaviour in brackish environments. Vegetatio 61: 189-195.

Scherer, K.Z. \& Castellani, T.T. 2004. Ecologia populacional de Paepalanthus polyanthus (Bong.) Kunth: variação temporal da distribuição espacial. Biotemas 17: 27-45.

Schmidt, I.B.; Figueiredo, I.B. \& Scariot, A. 2007. Ethnobotany and effects of harvesting on the population ecology of Syngonanthus nitens (Bong.) Ruhland (Eriocaulaceae), a NTFP from Jalapão Region, Central Brazil. Economic Botany 61: 73-85.

Slade, A. \& Hutchings, M.J. 1987. The effects of nutrients availability on foraging in the clonal herbs Glechoma hederacea. Journal of Ecology 75: $95-112$.

Song, M. \& Dong, M. 2002. Clonal plants and plant species diversity in wetland ecosystems in China. Journal of Vegetation Science 13: 237-244.

Suzuki, J. \& Stuefer, J.F. 1999. On the ecological and evolutionary significance of storage in clonal plants. Plants Species Biology 14: 11-17.

van Groenendael, J.M.; Klimes, L.; Klimesová, J. \& Hendriks, R.J.J. 1996. Comparative ecology of clonal plants. Philosophical Transactions of the Royal Society of London B 351: 1331-1339.

Walter, H. 1986. Vegetação e zonas climáticas: Tratado de ecologia global. São Paulo, Editora Pedagógica e Universitária.

Watkinson, A.R. \& White, J. 1985. Some life-history consequences of modular construction in plants. Philosophical Transactions of the Royal Society of London B 351: 31-51.

Zar, J.H. 1999. Bioestatistical analysis. Upper Saddle River, Prentice-Hall. 